\title{
INTEGRACIÓN DEL BALANCE HÍDRICO EN LA MODELACIÓN DE LA DISTRIBUCIÓN DE ESPECIES DE ÁRBOLES MEXICANOS
}

\author{
Raúl A. VACA ${ }^{1,3}$ y Duncan J. GOliCher ${ }^{1,2}$ \\ ${ }^{1}$ Departamento de Ecología y Sistemática Terrestre, División de la Conservación de la Biodiversidad, \\ El Colegio de la Frontera Sur, San Cristóbal de Las Casas, Chiapas, México \\ ${ }^{2}$ Centre for Conservation Ecology \& Environmental Change, School of Conservation Sciences, \\ Bournemouth University, Poole, Dorset, Reino Unido \\ ${ }^{3}$ Autor para la correspondencia: rgenuit@ecosur.mx
}

\begin{abstract}
Resumen: El modelado de la distribución de especies se basa típicamente en modelos estáticos que integran variables puramente climáticas. Una alternativa es utilizar modelos mecanísticos o semi-mecanísticos que incorporan variables que afectan la respuesta fisiológica de los organismos. En este trabajo demostramos la importancia de complementar el uso de estos dos tipos de modelos para predecir la distribución de especies de árboles mexicanos. Se seleccionaron 258 especies asociadas a cinco diferentes ecosistemas, que varían en su grado de exposición a los períodos de sequía y se ajustaron modelos aditivos generalizados (GAM). Se mapeó la dinámica anual de humedad del suelo $\left(\mathrm{DH}_{\text {anual }}\right)$ a lo largo de Mesoamérica, y se comparó la habilidad predictiva de este proceso en contraste con modelos ajustados con precipitación anual $\left(\mathrm{P}_{\text {anual }}\right)$. El efecto de la temperatura promedio y intervalo anual de temperatura se incluyó en ambos modelos. $\mathrm{DH}_{\text {anual }}$ mostró una capacidad significativamente mayor que $\mathrm{P}_{\text {anual }}$ para predecir la distribución de especies que presentan una respuesta general al estrés hídrico caracterizada por el ahorro y la tolerancia. Se observó un incremento promedio en el AUC al reemplazar $\mathrm{P}_{\text {anual }}$ por $\mathrm{DH}_{\text {anual }}$ para los grupos de especies de matorral xerófilo (0.15); de bosque tropical caducifolio (0.09) y de bosque tropical húmedo $(0.07)$. $\mathrm{DH}_{\text {anual }}$ no contribuyó a mejorar las predicciones para especies de áreas montañosas. El balance hídrico del suelo calculado a escala regional es un predictor valioso para entender diferencias en la distribución de especies de bosques tropicales cálidos de secos a húmedos, donde la temperatura se mantiene comparativamente más constante. Incorporar elementos espaciales, específicamente elevación, podría contribuir a definir mejor la distribución de especies de áreas montañosas. $\mathrm{DH}_{\text {anual }}$ introduce una importante fuente de incertidumbre respecto a la información climática debido a la dificultad de integrar parámetros del suelo y la variabilidad geomorfológica que ocurre a escalas locales.
\end{abstract}

Palabras claves: balance hídrico del suelo; modelo de cubeta; modelos aditivos generalizados; modelos de distribución de especies.

\begin{abstract}
Species distribution models are typically based on relationships between species occurrence and simple climatic variables. An alternative strategy is to use mechanistic or semi-mechanistic models that incorporate variables that are known to have important effects on physiological responses of the organisms. We compared models built using simple climatic variables with models incorporating calculations of monthly soil water balance when predicting the distribution of 258 Mexican tree species associated with five different ecosystems. The species chosen varied in their degree of exposure to periods of drought. Water balance was calculated using a bucket model that integrated the effects of evapotranspiration and rainfall over a year. Models incorporating calculated soil water balance were contrasted with models that used net annual rainfall as a measure of variability in water availability. The effects of mean annual temperature and temperature range were included in both sets of models. More detailed water balance models showed a significantly improved ability to predict the distribution of some, but not all, species. The observed increase in mean AUC when replacing $\mathrm{P}_{\text {anual }}$ by $\mathrm{DH}_{\text {anual }}$ was higher for the group of species of xeric scrub (0.15), intermediate for the group of species of deciduous tropical forests $(0.09)$, and significant, but comparatively low for the group of species of tropical moist forests (0.07). Calculated water balance did not improve predictions for species found in montane forests. We conclude that including detailed calculations of soil water balance can improve the species distribution models in areas of both moist and dry warm tropical forests, where temperature remains comparatively constant. However including variability in soil water balance is less important when species distributions are limited by the effect of elevation on temperature. Model predictions could be further refined through the incorporation of soil parameters and geomorphological variability at a local scales.
\end{abstract}

Keywords: bucket model, generalized additive models, water balance, species distribution models. 
L os modelos de distribución de especies tienen el propósito de determinar y mapear componentes del nicho ecológico de las especies en el espacio y a lo largo del tiempo (Guisan y Zimmermann, 2000; Guisan y Thuiller, 2005; Mateo et al., 2011). Las variables predictivas que se utilizan en los modelos, se espera que definan los factores que delimitan las condiciones favorables para la presencia de las especies. La selección de variables predictivas es de gran importancia puesto que de ello depende la validez de la interpretación de los modelos y, por consiguiente, la contribución de éstos al conocimiento de los mecanismos relacionados a los patrones de distribución de las especies y a la precisión en la tipificación de los nichos ecológicos. Idealmente, la selección de variables predictivas debería estar orientada a la incorporación de procesos ecológicos claves que afectan la distribución de las especies de manera directa (Guisan y Thuiller, 2005; Araújo y Guisan, 2006; Thuiller et al., 2008). Las variables predictivas representan gradientes directos cuando constituyen variables que tienen alguna influencia sobre la fisiología de los organismos, como por ejemplo nutrientes o la disponibilidad de agua en el suelo. Por el contrario, representan gradientes indirectos cuando no tienen una relación directa con la fisiología de éstos, pero tienen una fuerte correlación con los gradientes directos (Guisan y Zimmermann, 2000).

Austin et al. (2006) y Austin (2007) han argumentado que el uso de predictores que tienen una relación directa con la respuesta de las especies es importante para poder vincular la modelación estadística con la teoría ecológica, pudiendo también aumentar la capacidad de predicción de los modelos. La modelación de nichos ecológicos y áreas de distribución, sin embargo, se basa típicamente en variables puramente climáticas que no necesariamente representan los gradientes ambientales relacionados de manera directa con la distribución de las especies (Austin y Van Niel, 2011). Parámetros como temperatura promedio, precipitación anual, valores mensuales de precipitación o máximos y mínimos mensuales de temperatura, se asume muchas veces que están ligados de manera directa con los factores causales de la distribución de las especies; aunque ésto raramente sea puesto a prueba (Franklin, 2010a; Austin y Van Niel, 2011). Dado el efecto directo o indirecto del sustrato (material parental y suelo) en la disponibilidad de nutrientes y agua, los mapas de geología y suelos son también utilizados con frecuencia como variables predictivas en la modelación de nichos ecológicos (Franklin, 2010a). Todas estas variables son seleccionadas muchas veces por su simplicidad o por ser las únicas disponibles (Synes y Osborne, 2011). Sin embargo, cuando la verdadera distribución de una especie es incierta y los datos disponibles no son suficientes para tipificar el espacio actual del nicho ecológico, la interpretación de modelos basados en variables sustitutas de los factores que realmente afectan la distribución de los organismos puede resultar particularmente difícil de justificar (Soberón y Peterson, 2005; Pearson et al., 2006; Franklin, 2010b). Dadas las limitaciones de esta aproximación, los resultados obtenidos pueden ser imprecisos y varían entre modelos (Thuiller et al., 2004; Akcakaya et al., 2006; Botkin et al., 2007). La disponibilidad de conjuntos de datos climáticos como Worldclim o UNIATMOS-UNAM (para México, Centroamérica y Sur de EUA), ha supuesto un avance muy importante para el desarrollo de modelos de distribución. Como solución parcial, algunas de estas bases de datos proporcionan una serie de variables 'bio-climáticas' que permiten aumentar la interpretabilidad de los modelos generados respecto a los procesos que afectan a los organismos (Hijmans y Graham, 2006). Sin embargo, dichas variables han sido derivadas a partir de ecuaciones que combinan variables climáticas usando reglas sencillas, por lo que continúan siendo imprecisas para reflejar de manera verdadera los procesos que afectan su distribución (Piedallu et al., 2013). Existe, por otra parte, abundante conocimiento sobre los procesos eco-fisiológicos y biofísicos que gobiernan las relaciones entre las especies y el medioambiente, que puede ser utilizado para seleccionar variables potenciales que verdaderamente describan la distribución de las especies (Guisan y Zimmermann, 2000; Austin, 2007). Modelos mecanísticos o de procesos intentan representar matemáticamente los procesos fisiológicos y biológicos que traducen un factor físico o ambiental en una respuesta. En tales casos se construyen ecuaciones que intentan modelar la respuesta de una especie bajo distintos niveles de esos factores. Los modelos estáticos o correlativos, por el contrario, sólo construyen expresiones estadísticas que mejor puedan explicar la relación entre los lugares donde una especie está presente y los factores ambientales en esos lugares (Kearney y Porter, 2009; Buckley et al., 2010).

Un factor determinante para el desarrollo y la sobrevivencia de muchas especies de plantas es la duración de la estación de crecimiento. En áreas templadas, la estación de crecimiento es parcialmente determinada por la temperatura y la radiación solar. En los trópicos, en cambio, la estación de crecimiento está limitada principalmente por la disponibilidad de agua. Los efectos eco-fisiológicos del estrés hídrico sobre las plantas han sido extensamente documentados, incluyendo disminución en la transpiración e inhibición de la fotosíntesis, pudiendo resultar en la muerte de éstas si las sequías son severas o frecuentes (Reichstein et al., 2007). Si bien la relación entre la disponibilidad de agua y la distribución de la vegetación ha sido reconocida desde hace décadas (Stephenson, 1990), el balance hídrico en los suelos es raramente utilizado para modelar la distribución geográfica de las especies vegetales a una escala regional (Piedallu et al., 2013). Algunos estudios recientes han mostrado la utilidad de caracterizar la respuesta de las plantas al contenido de agua en los suelos (Lebourgeois et al., 2005; Engelbrecht et al., 2007; Granier et al., 2007; Lutz et al., 2010). En dichos estudios, la respuesta individual de las plantas a la disponi- 
bilidad de agua ha sido identificada como determinante de la diferenciación de nicho.

A pesar del gran avance en el desarrollo de técnicas y métodos para generar información espacial relacionada a procesos climáticos a escalas globales, la disponibilidad de descriptores ecológicos que influyen en la fisiología de las plantas es muy limitada (Synes y Osborne, 2011). Calcular el balance hídrico del suelo requiere de información que puede ser difícil de obtener. Medidas empíricas de evapotranspiración potencial pueden obtenerse a partir de datos generados por estaciones climáticas, sin embargo la información proporcionada por estos instrumentos resulta por lo general notoriamente inconsistente. Esta es la razón por la cual no existen capas de evapotranspiración disponibles en bases de datos como la de Worldclim (aunque medidas simples y empíricas de evapotranspiración potencial pueden generarse a partir de éstas). Como resultado, los ecólogos se inclinan generalmente por utilizar índices simples derivados directamente del patrón anual de precipitación (Piedallu et al., 2013). Por ejemplo, una regla convencional típicamente utilizada es que la precipitación excede la evapotranspiración durante meses con más de $100 \mathrm{~mm}$ de lluvia. Este criterio ha sido utilizado para calcular la estación de crecimiento para las plantas y generar modelos de distribución de especies (Golicher et al., 2012). Este criterio, sin embargo, utiliza una regla sencilla, lo cual es sensible a una decisión arbitraria sobre la cantidad de precipitación mensual necesaria para el crecimiento. Además la precipitación es un proceso estrictamente físico; mientras que la evapotranspiración está sujeta a procesos eco-fisiológicos y biológicos que son los que gobiernan las relaciones entre las especies y el medioambiente (Thornthwaite, 1948). Otra alternativa es utilizar NDVI (Normalizad Difference Vegetation Index), puesto que esta variable se encuentra correlacionada con productividad. Áreas con mayor NDVI presentan mayor transpiración que áreas donde el NDVI es menor. La respuesta general de las plantas al estrés hídrico se traduce en un uso conservativo de los recursos, caracterizado por una respuesta en general poco pronunciada y encaminada más al ahorro y la tolerancia que al gasto y la productividad (Valladares et al., 2008). Especies caducifolias, por ejemplo, adaptadas para sobrellevar la sequía, muestran una productividad baja o moderada en comparación con especies perennifolias. Sin embargo, NDVI se encuentra también afectado por el uso del suelo, integrando dos procesos en uno. Por lo tanto, si bien el NDVI representa una variable que puede ser considerada en la modelación de la distribución de especies, no es apto para construir un modelo basado exclusivamente en el clima.

La meta del presente trabajo fue mapear la dinámica anual de humedad del suelo a lo largo de Mesoamérica y comparar la habilidad de este proceso para describir el nicho ecológico de grupos de especies de árboles mexicanos con diferente grado de tolerancia a la sequía en relación a descriptores puramente climáticos, particularmente precipitación anual. Las metas específicas del trabajo fueron: (1) Evaluar si la capacidad predictiva de modelos incorporando el balance hídrico del suelo aumenta en comparación con modelos basados en precipitación anual, especialmente a medida que el factor hídrico contribuye más a explicar la distribución de las especies, (2) comparar la capacidad predictiva de modelos incorporando estas dos variables en relación a grupos de especies con diferentes intervalos de tolerancia a la sequía y (3) determinar la proporción de especies en los diferentes grupos cuya distribución no se logra predecir mejor a través del uso de índices de disponibilidad de agua en el suelo y explorar posibles razones por las cuales el uso de modelos mecanísticos contribuye a la tipificación del nicho ecológico de las especies. Con este trabajo se busca responder (1) si el balance hídrico del suelo calculado a escala regional es un predictor valioso para entender diferencias en la distribución de especies de zonas tropicales cálidas con diferente grado de tolerancia a la sequía y (2) si al considerar aspectos más mecanísticos en el proceso de modelación es posible captar mejor el nicho ecológico de especies cuya distribución está limitada mayormente por la disponibilidad de agua.

\section{Materiales y métodos}

Datos de las especies. Se seleccionaron 258 especies de árboles mexicanos, asociados a cinco diferentes ecosistemas que varían en su grado de exposición a los períodos de sequía, y abarcando un amplio intervalo de variación altitudinal. Se definieron cinco grupos de especies con base en la revisión de bibliografía y descripciones disponibles en sitios Web como Trópicos ${ }^{\circledast}$ (<http://www.tropicos.org/> consultado en agosto de 2012) y Missouri Botanical Garden (MOBOT) (<http://www.missouribotanicalgarden.org/> consultado en agosto de 2012). Tres grupos integran especies de ecosistemas de regiones tropicales cálidas: (1) Especies de bosques tropicales caducifolios (es decir, especies expuestas a períodos prolongados de déficit hídrico), (2) especies de matorrales xerófilos (es decir, especies expuestas a períodos más prolongados de déficit hídrico que las especies del primer grupo), (3) especies de bosques tropicales húmedos (es decir, especies expuestas a períodos cortos de sequía). Otros dos grupos integran especies de ecosistemas de regiones tropicales montañosas, incorporando variación altitudinal en la definición de grupos, (4) especies de bosques mesófilos de montaña y (5) especies de bosques de pino-encino y coníferas.

Se recopilaron datos de colectas botánicas para la región de América tropical, provenientes de tres fuentes diferentes de información: (1) La extensa base de datos del Global Biodiversity Information Facility (GBIF) (http://www.gbif. org/), (2) el Sistema Nacional de Información de Biodiversidad (SNIB) (http://www.conabio.gob.mx/remib/doctos/ snib.html) de la Comisión Nacional para el Conocimiento 
y Uso de la Biodiversidad (Conabio) (http://www.conabio. gob.mx/) y (3) la Red Internacional de Inventarios Forestales BIOTREE- NET (http://www.biotreenet.com/espanol/ $\mathrm{html} /$ ).

Los datos de las especies fueron agregados espacialmente a una resolución de $1 \times 1 \mathrm{~km}$ y se seleccionó, para cada especie, un dato único por cada celda independiente con información. Este procedimiento sirvió también como criterio de selección, considerándose únicamente especies para las cuales se contara con más de 20 datos independientes de presencia.

Variables predictivas. Se utilizaron cuatro variables predictivas relacionadas con temperatura y humedad, seleccionadas por poseer propiedades contrastantes y considerable grado de ortogonalidad. Tres de estas variables son de naturaleza puramente climática: (1) temperatura media anual (la cual se encuentra relacionada con elevación y con latitud), (2) intervalo anual de temperatura (que es una medida de estacionalidad y está determinada, hasta cierto punto, por la latitud) y (3) precipitación anual (relacionada con cualquier medida de duración y severidad de la estación seca). Estas tres variables se obtuvieron de la base de datos de WorldClim (<http://www.worldclim.org/> consultado en junio de 2012). La cuarta variable cuantifica el balance de agua en el suelo. Las plantas requieren del agua retenida en el suelo para sus diferentes procesos fisiológicos y biológicos; por lo tanto dicha variable representa un factor de crecimiento que puede controlar la distribución de las especies. El efecto de la temperatura se simplifica entonces al promedio anual y el intervalo; y se definen, por otra parte, una variable indirecta (es decir, precipitación anual) y otra directa (es decir, balance de agua en el suelo) relacionados con la disponibilidad de agua para las plantas.

La dinámica de humedad del suelo se calculó a partir de un modelo de cubeta, que toma como variables de entrada los mapas mensuales de precipitación de WorldClim y mapas mensuales de evapotranspiración potencial (ETP). Conociendo empíricamente el comportamiento de pérdida de agua del suelo, sin conocer todos los atributos y las complejas interrelaciones que lo gobiernan, se espera que el espacio del nicho ecológico de una especie sea previsible satisfactoriamente a partir de la estimación de la evapotranspiración, deficiencia y excedente hídricos; utilizando un modelo mecanístico. Para modelar la cantidad de agua que puede ser evaporada y transpirada dada la disponibilidad de agua en el suelo, se calcula la evapotranspiración real como una función del agua en el perfil de suelo y la evapotranspiración potencial.

La ecuación de Priestley-Taylor (Priestley y Taylor, 1972) es una de las más utilizadas en la actualidad para calcular ETP a escalas regionales o mayores. La forma más simple de esta ecuación considera temperatura y latitud como variables de entrada, aunque también funciona integrando la radiación solar neta. Es particularmente útil porque permite obtener estimaciones de la evapotranspiración potencial en ausencia de datos de humedad relativa y velocidad del viento, sustituyéndolos por un factor adimensional denominado "Coeficiente de Priestley-Taylor". La ecuación de Priestley-Taylor está implementada en el paquete EcoHydRology (Fuka et al., 2014) del lenguaje de programación para análisis estadístico R (R Development Core Team, 2008) y fue adaptada en este trabajo para calcular ETP utilizando las capas de información de 'Wordlclim'. Los datos de entrada utilizados incluyen: (1) las 36 capas de 'Worldclim' con información sobre temperaturas mínimas mensuales (12 capas), temperaturas máximas mensuales (12 capas) y precipitación mensual (12 capas); y (2) la latitud en radianes. A partir de los datos de entrada y la ecuación de PriestleyTaylor, se calcularon mapas mensuales de ETP (en mm/día) con una resolución espacial de $1 \times 1 \mathrm{~km}$. Las coberturas mensuales de ETP se encuentran disponibles en la siguiente dirección: http://geoserv.ecosur.mx/htmls/ModelosCubetaCapas.html[HT1]

Cálculo del balance hídrico del suelo. La evapotranspiración actual (ETA) es la cantidad de agua que puede ser evaporada y transpirada dada la disponibilidad de agua en el suelo. ETA se vuelve menor que ETP cuando la cantidad de humedad acumulada en el suelo decrece. Para modelar la dinámica de este proceso se usó un modelo de cubeta simplificado (Schaake et al., 1996). El contenido de agua en un perfil uniforme de suelo con una profundidad de un metro y porosidad de 0.5 , fue calculado mensualmente con base en la diferencia entre precipitación y ETP:

$$
\frac{d H}{d t}=P-E T A-P_{e}
$$

Donde $H$ representa la cantidad de agua en mm en un perfil de suelo uniforme, $P$ es la precipitación diaria, ETA es la evapotranspiración actual y $P_{e}$ es la escorrentía que ocurre cuando el perfil está saturado.

ETA es considerada como una función del agua en el perfil del suelo y la evapotranspiración potencial:

$$
\operatorname{ETA}=\operatorname{ETP}\left(1-\frac{H}{\left(H_{\text {sat }}-H_{p w p}\right)}\right)
$$

Donde $H$ es la cantidad de agua en el perfil, $H_{\text {sat }}$ es la cantidad de agua cuando el perfil está saturado y $H_{p w p}$ es la cantidad de agua en el punto de marchitez permanente.

El modelo no toma en cuenta la variabilidad en la profundidad ni en la porosidad de los suelos. El modelo en esta forma considera un valor de profundidad efectiva moderado y una porosidad moderada. Se justifica la omisión considerando la meta de producir un cálculo aproximado del balance hídrico a una escala regional. El efecto de la estructura del suelo afecta el balance hídrico a escala de cuencas, pero éste es difícil de incorporar en una forma consistente a esca- 
la regional dada la marcada variabilidad edáfica (sistemática y aleatoria) que puede ocurrir a escalas espaciales reducidas (Sharma y Luxmoore, 1979; Harrison et al., 1990). El modelo toma como variables de entrada los modelos mensuales de ETP generados, y los mapas mensuales de precipitación obtenidos de 'WorldClim'. Cada celda del área mapeada se considera como una cubeta individual no conectada con las que la rodean. La resolución temporal de la simulación es diaria. Para simular una resolución temporal diaria, el mapa mensual de precipitación del mes correspondiente se divide entre 30 días, y el valor resultante de precipitación se aplica a cada uno de los días del mes. En cada día de la simulación, se le suma a la cubeta el agua caída con la precipitación, y se le resta la pérdida por evapotranspiración. El día 30 de cada mes se monitorea el contenido de la cubeta, y se escribe en un mapa. La resolución espacial es de $1 \mathrm{~km}$. La dinámica anual de humedad del suelo se calcula, finalmente, como la sumatoria de los valores mensuales de contenido de agua en el suelo. Los modelos generados se encuentran disponibles en la siguiente dirección: http://geoserv.ecosur.mx/htmls/ ModelosCubetaCapas.html[HT2]

Modelos de distribución de especies. El ajuste de modelos de distribución se llevó a cabo utilizando modelos aditivos generalizados (GAM) con el paquete 'mgcv' (Wood, 2011) del lenguaje de programación para análisis estadístico R. Este método incorpora funciones no paramétricas que se adaptan mejor a estructuras no lineales de los datos. Se trata de modelos de regresión basados en polinomios continuos por intervalos (splines) que proporcionan una potente alternativa para introducir variables explicativas continuas con efectos no lineales sobre la variable dependiente. GAM es reconocido actualmente como un método versátil para la modelación de la distribución de especies (Guisan y Zimmermann, 2000; Thuiller, 2003; Segurado y Araújo, 2004; Elith y Leathwick, 2009). Modelos GAM requieren de datos de seudo-ausencias. Se generaron mil datos de seudo-ausencia de manera aleatoria siguiendo las recomendaciones de Barbet-Massin et al. (2012).

Para cada especie se ajustó: (1) Un modelo simple utilizando temperatura promedio anual, intervalo de temperatura y precipitación anual $\left(\mathrm{P}_{\text {anual }}\right)$. Los datos son datos binarios, por lo que se utilizan modelos GAM de la familia binomial. Sin embargo, como no hay interés en evaluar la significancia estadística de modelos ajustados a datos de seudo-ausencias (puesto que el tamaño de la muestra es arbitrario), se utilizan también modelos gausianos con el fin de simplificar la interpretación. (2) Un modelo GAM utilizando temperatura promedio anual, intervalo de temperatura anual y dinámica anual en la humedad del suelo $\left(\mathrm{DH}_{\text {anual }}\right)$. Este modelo es seguramente más realista puesto que el modelo de cubeta que determina la dinámica de humedad del suelo debe representar el patrón de estrés hídrico a lo largo del año.

El poder predictivo de $\mathrm{DH}_{\text {anual }}$ en relación a $\mathrm{P}_{\text {anual }}$, se eva- luó y comparó con cálculos del área debajo de la curva. Se ajustaron, de manera complementaria, modelos basados únicamente en las variables relacionadas con temperatura. El propósito de hacer ésto no se trata de comparar modelos ajustados con diferente número de variables predictivas, sino estimar la diferencia en el aporte proporcionado a cada modelo por $\mathrm{P}_{\text {anual }}$ en comparación con $\mathrm{DH}_{\text {anual }}$.

Idealmente la evaluación de modelos debería llevarse a cabo a partir de datos independientes los cuales son difíciles de obtener. Técnicas como la de validación cruzada y bootstrapping comparten el supuesto de que muestras seleccionadas al azar a partir de un conjunto de datos originales constituyen observaciones independientes y, por lo tanto, son adecuadas para la validación de modelos. Sin embargo, el supuesto de independencia no es cierto en el caso de datos espacialmente auto-correlacionados. De la misma manera, el problema de la auto-correlación espacial no se resuelve por aplicar remuestreo al azar del conjunto de datos originales, ni tampoco mediante la realización de muestreos adicionales dentro de la región modelada (Araújo et al., 2006). Por el contrario, una forma simple de evaluar la capacidad discriminatoria de modelos de distribución cuando no se cuenta con datos independientes, es dividiendo los datos espacialmente. Siguiendo este enfoque, se calcula la mediana de la longitud de todos los datos de presencia y se ajustan modelos a la mitad Este de la distribución. Estos modelos son utilizados para hacer predicciones en la porción Oeste. Luego se repite el proceso ajustando modelos a la mitad Oeste de la distribución y utilizándolos para hacer predicciones en la porción Este. Finalmente se selecciona para cada especie, el modelo asociado al proceso de división espacial que produce mejor ajuste.

\section{Resultados}

La Figura 1 compara el incremento en el AUC asociado a la incorporación de $\mathrm{P}_{\text {anual }}$ vs. $\mathrm{DH}_{\text {anual }}$ a modelos que integran temperatura anual promedio y intervalo anual de temperatura. El incremento promedio en el AUC para modelos que incluyen $\mathrm{P}_{\text {anual }}$ fue de 0.03 (valor promedio del AUC $=0.84$ ), mientras que para modelos que incluyen $\mathrm{DH}_{\text {anual }}$ fue de 0.07 (valor promedio del AUC $=0.89$ ). Los modelos basados en la dinámica de humedad del suelo mostraron tener un poder predictivo promedio significativamente más alto que aquellos basados en precipitación (promedio de la diferencia en el AUC, $+0.049, P<0.001$ ). Esto es predominantemente cierto a medida que la contribución del factor hídrico al rendimiento de los modelos es mayor. Es decir, a medida que el incremento en el AUC asociado al componente hídrico es mayor, $\mathrm{DH}_{\text {anual }}$ tiende sistemáticamente a ajustar mejores modelos que $\mathrm{P}_{\text {anual }}$ (Figura 1). En numerosos casos, el ajuste de modelos basados en $\mathrm{DH}_{\text {anual }}$ y $\mathrm{P}_{\text {anual }}$ fue similar. Esto ocurre principalmente cuando las variables asociadas con temperatura ajustan gran parte de la distribución de las especies (es 


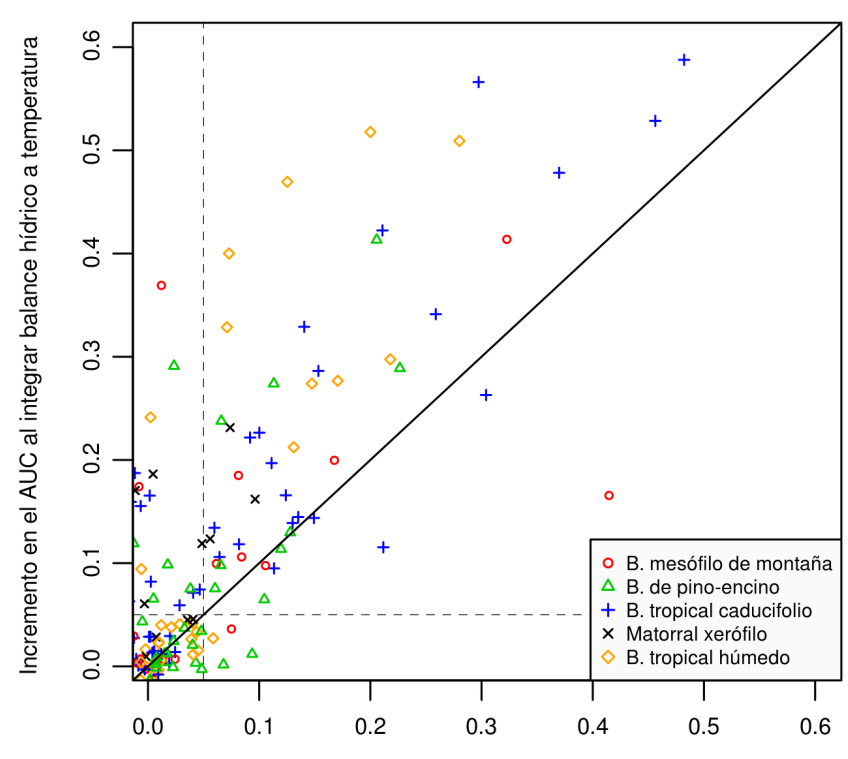

Incremento en el AUC al integrar precipitación anual a temperatura

Figura 1. Incremento en el valor AUC al agregar precipitación anual y dinámica anual de humedad en el suelo, a modelos de distribución de especies tropicales que consideran temperatura anual promedio y intervalo anual de temperatura ( $n=258$ especies). Los puntos por encima de la línea indican que la dinámica anual de humedad en el suelo ajusta mejores modelos que la precipitación anual. El incremento promedio en el AUC para modelos que incluyen precipitación anual fue de 0.03 (valor promedio del AUC $=0.84$ ), mientras que para modelos que incluyen balance hídrico anual fue de 0.07 (valor promedio del AUC = 0.89). El balance hídrico del suelo ajusta mejor que la precipitación para 84 de las 96 especies para las cuales el AUC se incrementó por un valor > 0.05 en relación a modelos ajustados únicamente con variables de temperatura.

decir, cuando el incremento en el AUC asociado al factor hídrico es < 0.05; ver la Figura 1).

Por otra parte, se examinó la capacidad predictiva de los índices de humedad para los grupos de especies definidos de acuerdo con su tolerancia a la sequía. Los modelos basados en la dinámica de humedad del suelo mostraron mejor ajuste que aquellos basados en precipitación, para los grupos de especies de bosque tropical caducifolio, bosque tropical peren- nifolio y matorrales xerófilos (Tabla 1). El detalle del ajuste de modelos especie por especies se presenta en el Anexo 1.

$\mathrm{DH}_{\text {anual }}$ mostró una capacidad significativamente mayor que $\mathrm{P}_{\text {anual }}$ para predecir la distribución de especies que presentan una respuesta general al estrés hídrico caracterizada por el ahorro y la tolerancia (Figura 2). Esto ocurrió para la totalidad de las especies asociadas a comunidades arbustivas de zonas áridas y semiáridas, con clima seco y lluvias escasas (es decir, matorrales xerófilos); así como también para la mayoría de las especies asociadas a comunidades arbóreas de clima cálido, con crecimiento en altura poco pronunciado y con una estación de secas y otra de lluvias muy marcadas a lo largo de año (i.e. bosques tropicales caducifolios) (Tabla 1). Para unas pocas especies asociadas al bosque tropical caducifolio, el ajuste de modelos basados en $\mathrm{DH}_{\text {anual }}$ fue similar que al considerar $\mathrm{P}_{\text {anual }}$. Esto ocurrió, específicamente, para diez especies del total de 64 especies que conformaron el grupo mencionado; tratándose de especies que en su mayoría pudieron ser identificadas como especies cuya distribución se encuentra asociada con factores no climáticos: (1) Acacia bilimekii J.F.Macbr. es una especie frecuente de vegetación de palmares, (2) Cameraria latifolia L. asociada a selvas bajas inundables e incluso manglares, (3) Eugenia laevis O.Berg. es típica de zapotales, (4) Heliocarpus palmeri S.Watson es una especie riparia, al igual que (5) Ficus membranacea C. Wright que se encuentra asociada a bosques de galería colindantes con el bosque tropical caducifolio y bosque tropical subcaducifolio, (6) Caesalpinia platyloba S. Watson crece en bosques secos, deciduos, en la línea de costa sobre terreno arenoso, (7) Mimosa eurycarpa B.L.Rob. está asociada a suelos de areniscas. Los modelos de distribución que se muestran en la Figura 3 son ejemplo de dos especies de bosques secos para las cuales el uso de $\mathrm{DH}_{\text {anual }}$ permitió ajustar mejores modelos que al usar $\mathrm{P}_{\text {anual }}$.

La capacidad predictiva de $\mathrm{DH}_{\text {anual }}$ fue también mayor que $\mathrm{P}_{\text {anual }}$ para el grupo de especies de bosques tropicales húmedos; sin embargo, la magnitud en el incremento promedio del AUC fue marcadamente menor en relación a los dos grupos anteriores. De la misma manera, la proporción de especies cuya distribución se explica mejor a partir de $\mathrm{DH}_{\text {anual }}$, fue marcadamente menor en relación a los grupos

Tabla 1. Capacidad de los índices de humedad para predecir la distribución de especies de árboles mexicanos agrupados según grado de tolerancia a la sequía $(n=258)$. Se muestra el promedio del AUC para modelos que integran balance anual de humedad en el suelo $\left(\mathrm{DH}_{\text {anual }}\right)$ y precipitación anual $\left(\mathrm{P}_{\text {anual }}\right)$, junto a temperatura anual promedio y intervalo anual de temperatura. Los modelos fueron comparados con pruebas $\mathrm{t}$ de Student. Se detalla el porcentaje de especies cuya distribución se predice mejor a partir de modelos que integran balance anual de humedad en el suelo.

\begin{tabular}{|c|c|c|c|c|c|c|c|}
\hline & $N$ & $\mathrm{DH}_{\text {anual }}$ & $\mathbf{P}_{\text {anual }}$ & $t$ & gl & $p$ & $\%$ de especies \\
\hline Bosque tropical caducifolio & 64 & 0.92 & 0.83 & 6.10 & 63 & $<0.001$ & 86 \\
\hline Matorral xerófilo & 29 & 0.92 & 0.77 & 7.88 & 28 & $<0.001$ & 100 \\
\hline Bosque tropical perennifolio & 52 & 0.90 & 0.83 & 2.79 & 51 & $<0.01$ & 58 \\
\hline Bosque mesófilo de montaña & 35 & 0.88 & 0.86 & 0.73 & 34 & 0.47 & 51 \\
\hline Bosque de pino-encino & 78 & 0.86 & 0.87 & -1.25 & 77 & 0.22 & 37 \\
\hline
\end{tabular}



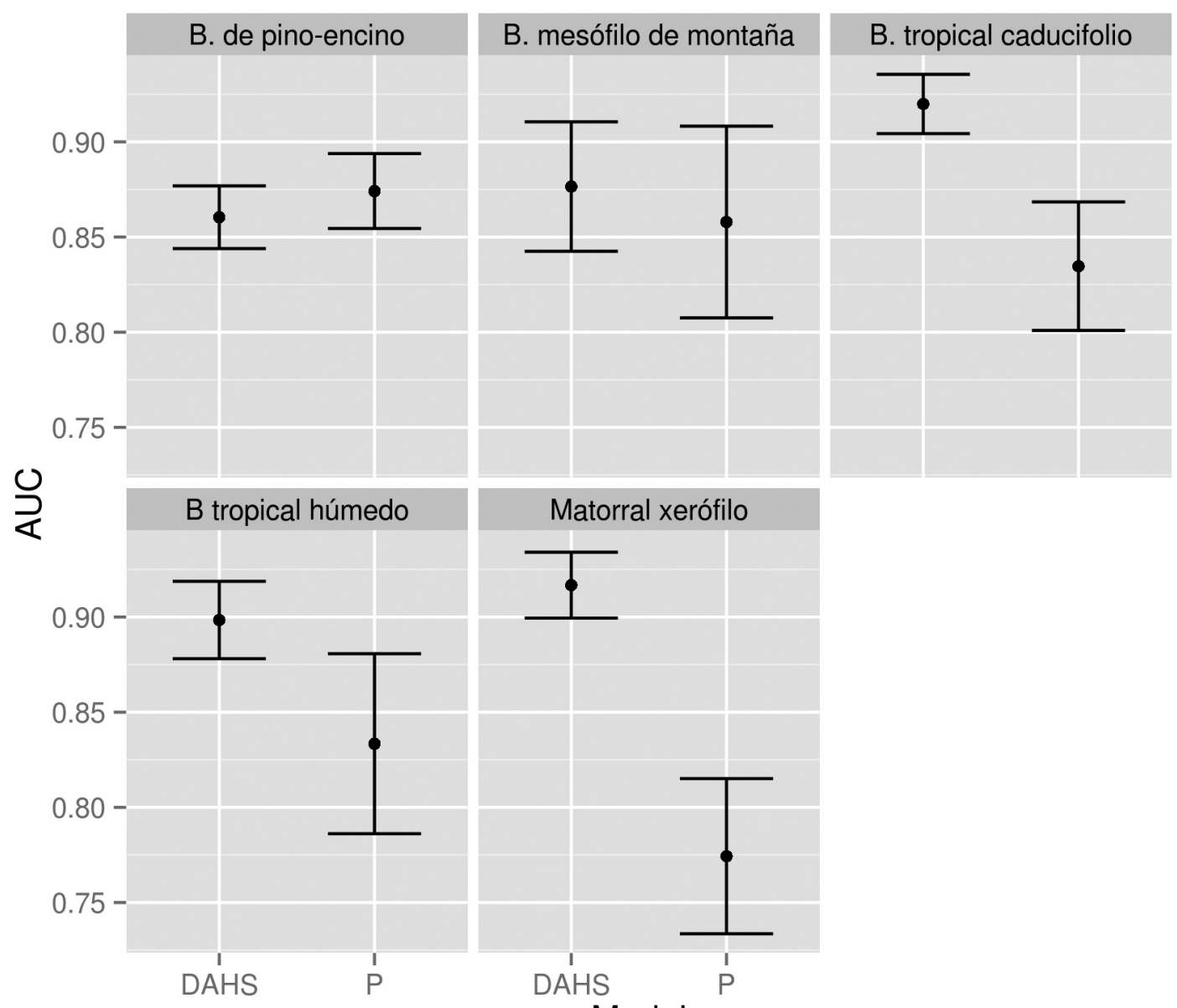

\section{Modelo}

Figura 2. AUC promedio e intervalos de confianza para modelos que integran balance anual de humedad en el suelo $\left(\mathrm{DH}_{\text {anual }}\right) \mathrm{y}_{\mathrm{precipita}-}$ ción anual $\left(\mathrm{P}_{\text {anual }}\right)$ junto a temperatura anual promedio y intervalo anual de temperatura, discriminado por grupos de especies según grado de tolerancia a la sequía.

de especies de bosques secos. En cuanto a los grupos de especies asociadas a bosques mesófilos de montaña y pinoencino, el ajuste de modelos basados en $\mathrm{DH}_{\text {anual }}$ no mostró ser mejor que el ajuste de modelos obtenido a partir de $\mathrm{P}_{\text {anual }}$. Los modelos obtenidos en tales casos pueden interpretarse en términos de una distribución mayormente asociada a la respuesta de las especies a la temperatura o del intervalo altitudinal preferido por las especies (dado que las variables asociadas a temperatura están altamente correlacionadas con elevación). Los modelos de distribución que se muestran en la Figura 4 son ejemplo de dos especies de bosques tropicales de montaña para las cuales el ajuste de modelos basados en $\mathrm{DH}_{\text {anual }}$ y $\mathrm{P}_{\text {anual }}$ fue similar. Los modelos de distribución generados para todas las especies, sumado a un diagnóstico detallado para cada modelo, pueden consultarse en la siguiente liga: http://geoserv.ecosur.mx.

\section{Discusión}

Los resultados obtenidos sugieren que el balance hídrico del suelo calculado al nivel regional es un predictor valio- so para entender diferencias en la distribución de especies de zonas tropicales cálidas con diferente grado de tolerancia a la sequía, aún sin la adición de detalles específicos de las características del suelo. En el presente trabajo se pone en evidencia que el uso de variables puramente climáticas como precipitación anual, puede ser inadecuado para representar la disponibilidad de agua en el suelo para las plantas. De acuerdo con Piedallu et al. (2013), variables climáticas simples relacionadas con precipitación pueden no traducirse en reserva de agua para las plantas cuando no hay humedad acumulada en el suelo, subestimando la importancia del agua para las mismas; de la misma manera puede sobreestimar el estrés hídrico cuando no llueve pero sí hay humedad acumulada en el suelo.

Se demostró, además, que cuando se consideran aspectos más mecanísticos en el proceso de modelación, es posible captar mejor la distribución de especies que responden mayormente al clima. Nuestros resultados muestran claramente que los proxies puramente climáticos pueden conducir a una subestimación de la importancia del agua en estudios de distribución de plantas; una conclusión que está de acuerdo con 

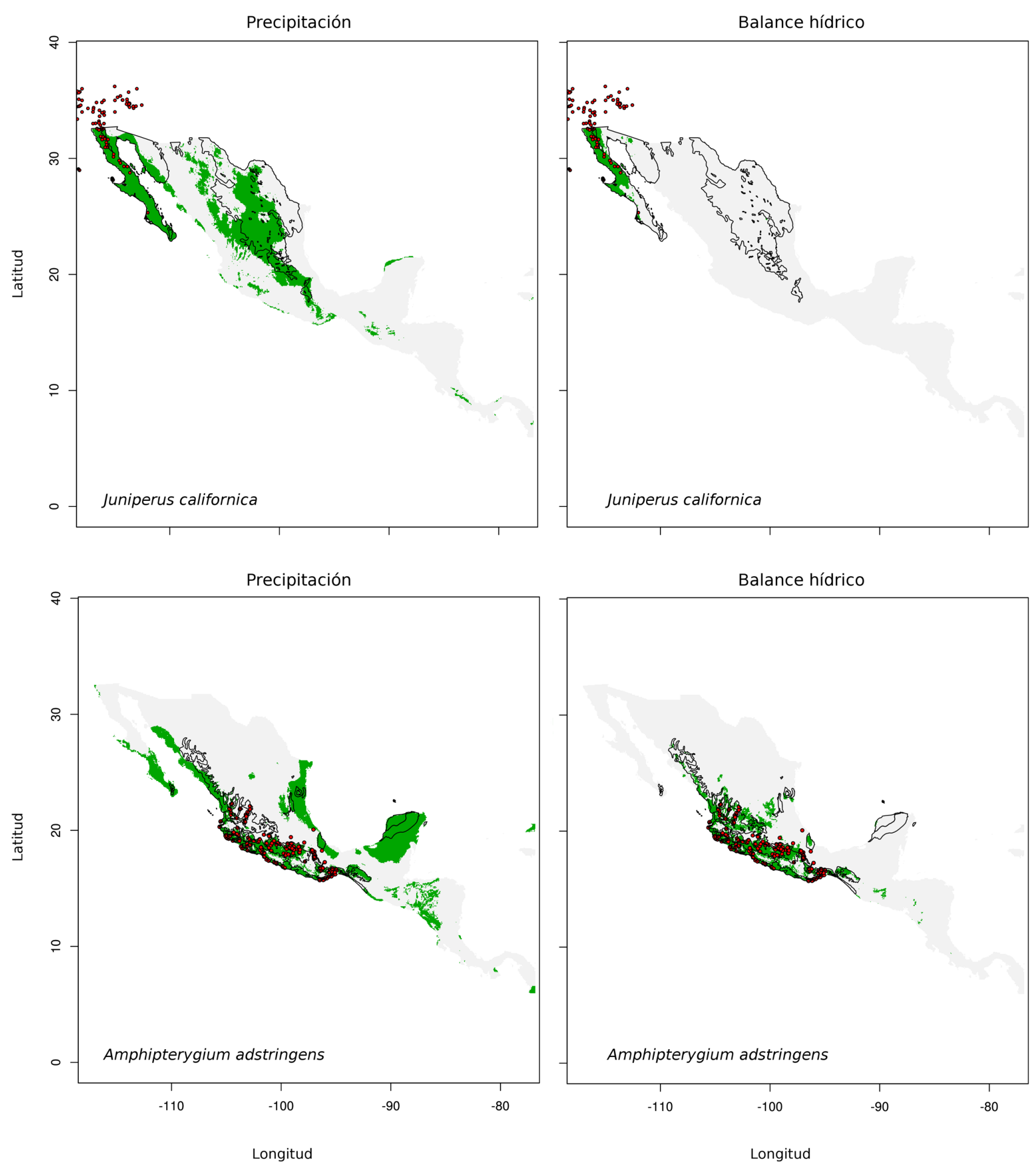

Figura 3. Diferencias en la distribución de dos especies de árboles de bosques secos, de acuerdo al ajuste de modelos basados en precipitación anual vs. balance hídrico anual. Juniperus californica es una especie de matorral xerófilo, mientras que Amphipterygium adstringens es característica del bosque tropical caducifolio. A modo de referencia, los mapas muestran la delimitación (contorno negro) del matorral xerófilo y del bosque tropical caducifolio/subcaducifolio en México de acuerdo con Rzedowski (1990), respectivamente. Ambos ejemplos representan casos en los cuales los modelos basados en el balance hídrico anual mostraron mejor ajuste que aquellos basados en precipitación anual (diferencia en el AUC para Juniperus californica: $\mathrm{P}_{\text {anual }}=0.70 v s . \mathrm{DH}_{\text {anual }}=0.96$; diferencia en el AUC para Amphipterygium adstringens: $\mathrm{P}_{\text {anual }}=0.80 \mathrm{vs} . \mathrm{DH}_{\text {anual }}=0.91$ ). Todos los modelos integran temperatura media anual y intervalo anual de temperatura. El balance hídrico parece mejorar el rendimiento de los modelos mediante el refinado de la predicción de la distribución de las especies en áreas con condiciones de precipitación similares pero donde existe variación en la distribución de la precipitación durante el año. 

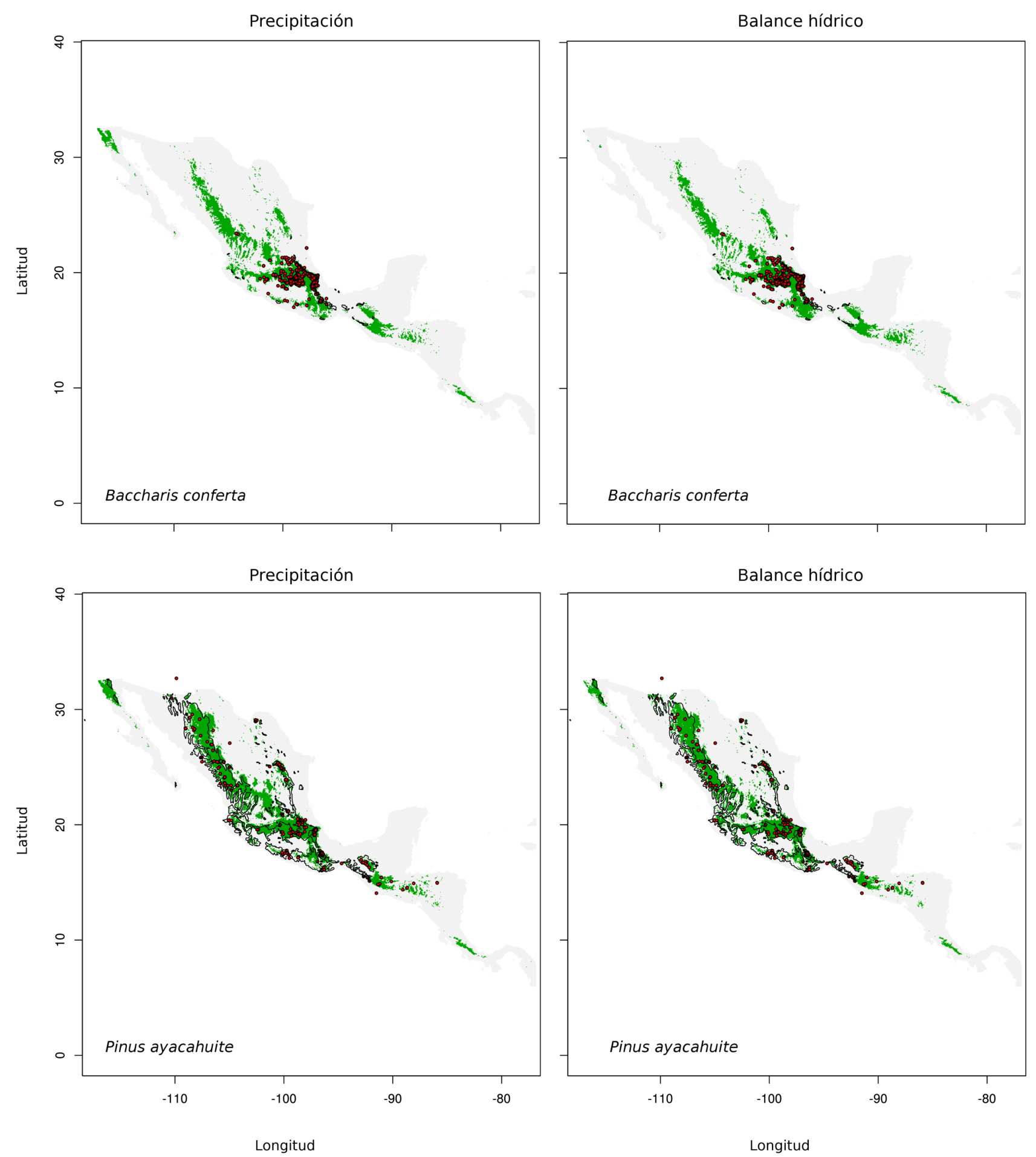

Figura 4. Diferencias en la distribución de dos especies de árboles de bosques tropicales de montaña, de acuerdo al ajuste de modelos basados en precipitación anual vs. balance hídrico anual. Baccharis conferta es una especie característica del bosque mesófilo de montaña, mientras que Pinus ayacahuite es característica del bosque de confieras y encinos. A modo de referencia, los mapas muestran la delimitación (contorno negro) del bosque mesófilo de montaña y del bosque de confieras y encinos en México de acuerdo con Rzedowski (1990), respectivamente. Ambos ejemplos representan casos en los cuales los modelos basados en el balance hídrico anual y en precipitación anual mostraron un ajuste similar (diferencia en el AUC para Baccharis conferta: $\mathrm{P}_{\text {anual }}=0.95$ vs $. \mathrm{DH}_{\text {anual }}=0.93$; diferencia en el AUC para Pinus ayacahuite: $\mathrm{P}_{\text {anual }}=$ 0.91 vs. $\mathrm{DH}_{\text {anual }}=0.89$ ). Todos los modelos integran temperatura media anual y intervalo anual de temperatura. Los modelos obtenidos pueden interpretarse en términos de una distribución mayormente asociada con la respuesta de las especies a la temperatura. 
numerosos estudios que demuestran el papel fundamental del balance hídrico del suelo en la fisiología de los árboles y el crecimiento (Lebourgeois et al., 2005; Granier et al., 2007). Por lo tanto, este trabajo proporciona información útil para entender la importancia del balance hídrico como determinante de la distribución de árboles tropicales.

La variable climática $\mathrm{P}_{\text {anual }}$ sigue siendo un índice apropiado para predecir la distribución de $60 \%$ de las especies de árboles estudiadas; en tales casos la capacidad predictiva de $\mathrm{P}_{\text {anual }}$ fue similar a la de $\mathrm{DH}_{\text {anual }}$. Estas especies fueron principalmente especies de bosques templados de áreas montañosas, cuya distribución está menos influenciada por la disponibilidad de agua. Su distribución parece estar influenciada principalmente por la temperatura; teniendo las variables hídricas estudiadas, poca influencia sobre su distribución. Esto permite que las diferentes variables asociadas al componente hídrico puedan ser utilizadas en los modelos de manera indistinta. En tales casos debería considerarse de manera explícita la incorporación de elementos espaciales en los modelos, específicamente elevación, lo cual podría mejorar los modelos predictivos dado que los intervalos altitudinales podrían contribuir a definir mejor la distribución de especies de áreas montañosas. Estos resultados complementan la investigación en áreas templadas montañosas, donde se identifica a la temperatura como un predictor apropiado y preciso de la distribución de las plantas (Rorison et al., 1986; Grace, 1987; Gottfried et al., 1999; Mckenzie et al., 2003; Morin et al., 2007).

Por el contrario, se demuestra que la disponibilidad de agua es un determinante más importante que la temperatura, en la determinación de la distribución de especies de árboles de regiones tropicales cálidas. Para especies de zonas tropicales cálidas, el balance hídrico del suelo se comporta mejor que los proxies climáticos. En este sentido, el mayor efecto de la disponibilidad de agua en comparación con otros predictores climáticos simples ha sido previamente reportado (Piedallu et al., 2013). En bosques tropicales caducifolios y matorrales xerófilos donde es común que las plantas queden expuestas a períodos de estrés hídrico, dominan las especies caducifolias adaptadas para minimizar la pérdida de agua durante los períodos de baja disponibilidad. Modelos con una perspectiva más mecanística, que incorporan procesos como el balance hídrico, representan de manera más adecuada la disponibilidad de agua en el suelo para las plantas y permiten obtener mejores predicciones de la distribución de este tipo de especies. Para diversas especies de bosques tropicales húmedos, la capacidad predictiva de $\mathrm{P}_{\text {anual }}$ fue similar a la de $\mathrm{DH}_{\text {anual }}$. Cabe señalar, en este sentido, que los bosques tropicales húmedos pueden consistir o no, en bosques estacionales con temporadas cortas sin lluvias incluyendo especies de bosques subperennifolios a subcaducifolios y selvas altas a medianas, pudiendo de esta forma estar relacionados con bosques estacionales que presentan numerosas especies de árboles que pierden las hojas en la temporada de sequía. Es posible, entonces, que especies asociadas a ambientes más estacionales en cuanto a la disponibilidad de agua, sean las que tienden a mostrar un mejor ajuste a partir de $\mathrm{DH}_{\text {anual }}$. De la misma manera que especies asociadas a ambientes menos estacionales sean las que tienden a mostrar similar ajuste a partir de $\mathrm{P}_{\text {anual }}$ o $\mathrm{DH}_{\text {anual }}$

Cabe señalar, finalmente, que $\mathrm{DH}_{\text {anual }}$ introduce una importante fuente de incertidumbre respecto a la información climática debido a la dificultad de integrar los parámetros del suelo y la variabilidad espacial en el contenido de agua en el suelo a escalas locales debido a atributos geomorfológicos; como se ha observado en estudios a escala local (Lookingbill y Urban, 2004; Dyer, 2009). Por otra parte, la escorrentía tampoco ha sido incluida en el cálculo del balance hídrico, a pesar de la probabilidad que tiene de mejorar la calidad de las estimaciones (Redding y Devito, 2008). Las mejoras en el cálculo de balance hídrico del suelo pueden proporcionar un mayor conocimiento de la importancia de las variables de agua del suelo en los modelos de distribución en el futuro. En regiones tropicales, donde nuestra comprensión sobre la distribución geográfica de la mayoría de las especies se encuentra aún incompleta, el uso de este tipo de aproximaciones podría contribuir a mejorar nuestra comprensión sobre los mecanismos relacionados a los patrones de distribución de las especies y permitir una tipificación más precisa de los nichos ecológicos. En el actual contexto de cambio climático, el uso de predictores relevantes es crucial para evaluar las consecuencias en términos de cambios en la vegetación. Estos predictores también pueden contribuir a la identificación de refugios u oportunidades potenciales para la expansión local en el contexto de la migración de las especies (Keppel et al., 2012).

El desarrollo de métodos eficientes para estimar el agua disponible en el suelo a escalas regionales o mayores, es un paso necesario e importante en la evaluación de los efectos del estrés hídrico en las plantas. El uso de mapas de dinámica de humedad del suelo mejora nuestra comprensión de los mecanismos relacionados con los patrones de distribución de las plantas y permite una mejor determinación de los nichos ecológicos de las especies. Mapas de tipos de suelo y geología podrían contribuir a definir mejores modelos de disponibilidad de agua. Sin embargo, el uso de mapas digitales de geología y suelos puede ser problemático puesto que este tipo de mapas han sido generados comúnmente utilizando una perspectiva cartográfica, la cual tiene un propósito principalmente comunicativo y el objetivo de proporcionar modelos meramente visuales (Goodchild, 1988; Demers, 1991). Sólo en décadas recientes se ha comenzado a elaborar mapas de geología y suelos con base en sensores remotos y modelos predictivos (Scull et al., 2003). La escala gruesa de este tipo de mapas, sin embargo, continúa dificultando su incorporación en la construcción de modelos de disponibilidad de agua en el suelo (Franklin, 2010a). 


\section{Agradecimientos}

Este trabajo fue financiado por la Comisión Nacional para el Conocimiento y Uso de la Biodiversidad (CONABIO). Número de proyecto FB1609/JM052/12. Estamos muy agradecidos a los dos revisores anónimos cuyos comentarios fueron sumamente útiles para mejorar la claridad y coherencia del manuscrito.

\section{Literatura citada}

Akçakaya H.R., Butchart S.H.M., Mace G.M., Stuart S.N. y Hilton-Taylor C. 2006. Use and misuse of the IUCN Red List Criteria in projecting climate change impacts on biodiversity. Global Change Biology 12:2037-2043.

Araújo M.B. y Guisan A. 2006. Five (or so) challenges for species distribution modelling. Journal of Biogeography 33:16771688.

Austin M.P., Belbin L., Meyers J.A., Doherty M.D. y Luoto M. 2006. Evaluation of statistical models used for predicting plant species distributions: role of artificial data and theory. Ecological Modelling 199:197-216.

Austin M. 2007. Species distribution models and ecological theory: A critical assessment and some possible new approaches. Ecological Modelling 200:1-19.

Austin M.P. y Van Niel K.P. 2011. Improving species distribution models for climate change studies: variable selection and scale. Journal of Biogeography 38:1-8.

Barbet-Massin M., Jiguet F., Albert C.H. y Thuiller W. 2012. Selecting pseudo-absences for species distribution models: how, where and how many? Methods in Ecology and Evolution 3:327-338.

Botkin D.B., Saxe H., Araújo M.B., Betts R., Bradshaw R.H.W., Cedhagen T., Chesson P., Dawson T.P., Etterson J.R., Faith D.P., Ferrier S., Guisan A., Hansen A.S., Hilbert D.W., Loehle C., Margules C., New M., Sobel M.J. y Stockwell D.R.B. 2007. Forecasting the effects of global warming on biodiversity. BioScience 57:227-236.

Buckley L.B., Urban M.C., Angilletta M.J., Crozier L.G., Rissler L.J. y Sears M.W. 2010. Can mechanism inform species' distribution models? Ecology Letters 13:1041-1054.

Demers M.N. 1991. Classification and purpose in automated vegetation maps. Geographical Review 81:267-280.

Dyer J.M. 2009. Assessing topographic patterns in moisture use and stress using a water balance approach. Landscape Ecology 24:391-403.

Elith J. y Leathwick J.R. 2009. Species distribution models: Ecological explanation and prediction across space and time. Annual Review of Ecology, Evolution and Systematics 40:677-697.

Engelbrecht B.M.J., Comita L.S., Condit R., Kursar T.A., Tyree M.T., Turner B.L. y Hubbell S.P. 2007. Drought sensitivity shapes species distribution patterns in tropical forests. Nature 447:80-82.

Franklin J. 2010a. Mapping Species Distributions: Spatial Inference and Prediction. Cambridge University Press, Nueva York.

Franklin J. 2010b. Moving beyond static species distribution models in support of conservation biogeography. Diversity and Distributions 16:321-330.

Fuka D.R., Walter M.T., Archibald J.A., Steenhuis T.S. y Easton
Z.M. 2014. EcoHydRology: A community modeling foundation for Eco-Hydrology. R package version 0.4.12. http://CRAN.Rproject.org/package=EcoHydRology

Golicher D.J., Cayuela L. y Newton A.C. 2012. Effects of climate change on the potential species richness of Mesoamerican forests. Biotropica 44:284-293.

Goodchild M.F. 1988. Stepping over the line: Technological constraints and the new cartography. American Cartographer 15:311-319.

Gottfried M., Pauli H., Reiter K. y Grabherr G. 1999. A fine-scaled predictive model for changes in species distribution patterns of high mountain plants induced by climate warming. Diversity and Distributions 5:241-251.

Grace J. 1987. Climtatic tolerance and the distribution of plants. New Phytologist 106:113-130.

Granier A., Reichstein M., Bréda N., Janssens I.A., Falge E., Ciais P., Grünwald T., Aubinet M., Berbigier P., Bernhofer C., Buchmann N., Facini O., Grassi G., Heinesch B., Ilvesniemi H., Keronen P., Knohl A., Köstner B., Lindroth A., Longdoz B., Loustau D., Mateus J., Montagnani L., Nys C., Moors E., Papale D., Peiffer M., Pilegaard K., Pita G., Pumpanen J., Rambal S., Rebmann C., Rodrigues A., Seufert G., Tenhunen J., Vesala T. y Wang Q. 2007. Evidence for soil water control on carbon and water dynamics in European forests during the extremely dry year: 2003. Agricultural and Forest Meteorology 143:123-145.

Guisan A. y Thuiller W. 2005. Predicting species distribution: offering more than simple habitat models. Ecology Letters 8:9931009.

Guisan A. y Zimmermann N.E. 2000. Predictive habitat distribution models in ecology. Ecological Modelling 135:147-186.

Harrison J.B.J., McFadden L.D. y Weldon R.J. 1990. Spatial soil variability in the Cajon Pass chronosequence: Implications for the use of soils as a geochronological tool. Geomorpholgy 3:399-416.

Hijmans R.J. y Graham C.H. 2006. The ability of climate envelope models to predict the effect of climate change on species distributions. Global Change Biology 12:2272-2281.

Kearney M. y Porter W. 2009. Mechanistic niche modelling: combining physiological and spatial data to predict species' ranges. Ecology Letters 12:334-350.

Keppel G., Van Niel K.P., Wardell-Johnson G.W., Yates C.J., Byrne M., Mucina L., Schut A.G.T., Hopper S.D. y Franklin S.E. 2012. Refugia: identifying and understanding safe havens for biodiversity under climate change. Global Ecology and Biogeography 21:393-404.

Lebourgeois F., Bréda N., Uldrich E. y Granier A. 2005. Climatetree-growth relationships of European beech (Fagus sylvatica L.) in the French Permanent Plot Network (RENECOFOR). Trees 19:385-401.

Lookingbill T. y Urban D. 2004. An empirical approach towards improved spatial estimates of soil moisture for vegetation analysis. Landscape Ecology 19:417-433.

Lutz J.A., van Wagtendonk J.W. y Franklin J.F. 2010. Climatic water deficit, tree species ranges, and climate change in Yosemite National Park. Journal of Biogeography 37:936-950.

Mateo R.G., Felicísimo Á.M. y Muñoz J. 2011. Modelos de distribución de especies: Una revisión sintética. Revista Chilena de Historia Natural 84:217-240.

McKenzie D., Peterson D.W., Peterson D.L. y Thornton P.E. 2003. 
Climatic and biophysical controls on conifer species distributions in mountain forests of Washington State, USA. Journal of Biogeography 30:1093-1108.

Morin X., Augspurger C. y Chuine I. 2007. Process-based modeling of species' distributions: What limits temperate tree species' range boundaries? Ecology 88:2280-2291.

Pearson R.G., Thuiller W., Araújo M.B., Martinez-Meyer E., Brotons L., McClean C., Miles L., Segurado P., Dawson T.P. y Lees D.C. 2006. Model-based uncertainty in species range prediction. Journal of Biogeography 33:1704-1711.

Piedallu C., Gégout J.C., Perez V. y Lebourgeois F. 2013. Soil water balance performs better than climatic water variables in tree species distribution modelling. Global Ecology and Biogeography 22:470-482.

Priestley C.H.B. y Taylor R.J. 1972. On the assessment of surface heat flux and evaporation using large-scale parameters. Monthly Weather Review 100:81-92.

R Development Core Team. 2008. R: A language and environment for statistical computing. R Foundation for Statistical Computing, Vienna.

Redding T.E. y Devito K.J. 2008. Lateral flow thresholds for aspen forested hillslopes on the Western Boreal Plain, Alberta, Canada. Hydrological Processes 22:4287-4300.

Reichstein M., Ciais P., Papale D., Valentini R., Running S., Viovy N., Cramer W., Granier A., Ogée J., Allard V., Aubinet M., Bernhofer Chr., Buchmann N., Carrara A., Grünwald T., Heimann M., Heinesch B., Knohl A., Kutsch W., Loustau D., Manca G., Matteucci G., Miglietta F., Ourcival J.M., Pilegaard K., Pumpanen J., Rambal S., Schaphoff S., Seufert G., Soussana J.-F., Sanz M.-J., Vesala T. y Zhao M. 2007. Reduction of ecosystem productivity and respiration during the European summer 2003 climate anomaly: a joint flux tower, remote sensing and modelling analysis. Global Change Biology, 13:634-651.

Rorison I.H., Sutton F. y Hunt R. 1986. Local climate, topography and plant growth in Lathkill Dale NNR . I . A twelve-year summary of solar radiation and temperature. Plant, Cell and Environment 9:49-56.

Schaake J.C., Koren V.I. y Duan Q.Y. 1996. Simple water balance model for estimating runoff at different spatial and temporal scales. Journal of Geophysical Research 101:7461-7475.

Scull P., Franklin J., Chadwick O.A. y McArthur D. 2003. Predic- tive soil mapping: a review. Progress in Physical Geography 27:171-197.

Segurado P. y Araújo M.B. 2004. An evaluation of methods for modelling species distributions. Journal of Biogeography 31:1555-1568.

Sharma M.L. y Luxmoore R.J. 1979. Soil spatial variability and its consequences on simulated water balance. Water Resources Research 15:1567-1573.

Soberón J. y Peterson A.T. 2005. Interpretation of models of fundamental ecological niches and species' distributional areas. Biodiversity Informatics 2:1-10.

Stephenson N.L. 1990. Climatic control of vegetation distribution: The role of the water balance. American Naturalist 135:649670.

Synes N.W. y Osborne P.E. 2011. Choice of predictor variables as a source of uncertainty in continental-scale species distribution modelling under climate change. Global Ecology and Biogeography 20:904-914.

Thornthwaite C.W. 1948. An approach toward a rational classification of climate. Geographical Review 38:55-94.

Thuiller W., Araújo M.B., Pearson R.G., Whittaker R.J., Brotons L. y Lavorel S. 2004. Biodiversity conservation: Uncertainty in predictions of extinction risk. Nature 430:145-148.

Thuiller W., Albert C., Araújo M.B., Berry P.M., Cabeza M., Guisan A., Hickler T., Midgley G.F., Paterson J., Schurr F.M., Sykes M.T. y Zimmermann N.E. 2008. Predicting global change impacts on plant species' distributions: Future challenges. Perspectives in Plant Ecology, Evolution and Systematics 9:137-152.

Thuiller W. 2003. BIOMOD - optimizing predictions of species distributions and projecting potential future shifts under global change. Global Change Biology 9:1353-1362.

Valladares F., Vilagrosa A., Peñuelas J., Ogaya R. Camarero J.J., Corcuera L, Sisó S y Gil-Pelegrín E. 2004. Estrés hídrico: ecofisiología y escalas de la sequía. En: Valladares F. Ed. Ecología del bosque mediterráneo en un mundo cambiante, pp. 163-190, Ministerio de Medio Ambiente, EGRAF S.A., Madrid.

Wood S.N. 2011. Fast stable restricted maximum likelihood and marginal likelihood estimation of semiparametric generalized linear models. Journal of the Royal Statistical Society (Series B) 73:3-36.

Recibido: 8 de septiembre de 2014

Aceptado: 10 de enero de 2015 
Anexo 1. Resultados del ajuste de modelos aditivos generalizados (GAM) especie por especie. Se incluyen los resultados de los modelos basados únicamente en variables relacionadas a temperatura (GAM_T: temperatura promedio anual, intervalo de

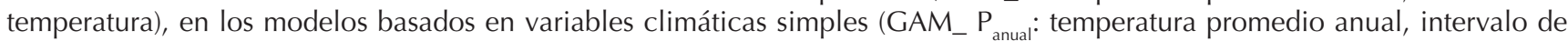
temperatura y precipitación anual) y en los modelos reemplazando precipitación anual por dinámica anual en la humedad del suelo $\left(\mathrm{GAM} \_\mathrm{DH}_{\mathrm{anual}}\right)$. Los resultados corresponden a cálculos del área debajo de la curva (AUC).

\begin{tabular}{|c|c|c|c|c|}
\hline Especie & Autoridad & GAM_T & GAM_P anual $_{\text {a }}$ & GAM_DH ${ }_{\text {anual }}$ \\
\hline Abatia mexicana & Standl. & 0.69571 & 0.68763 & 0.86989 \\
\hline Acacia bilimekii & J.F. Macbr. & 0.94626 & 0.96532 & 0.94932 \\
\hline Adelobotrys adscendens & (Sw.) Triana & 0.44755 & 0.64755 & 0.96533 \\
\hline Aegiphila falcata & Donn. Sm. & 0.90405 & 0.96952 & 0.82316 \\
\hline Alfaroa costaricensis & Standl. & 0.96295 & 0.97335 & 0.97518 \\
\hline Amphipterygium adstringens & (Schltdl.) Standl. & 0.43605 & 0.80578 & 0.91430 \\
\hline Arachnothryx buddleioides & (Benth.) Planch. & 0.91992 & 0.91321 & 0.90682 \\
\hline Arachnothryx manantlanensis & (Lorence) Borhidi & 0.68136 & 0.48232 & 0.80420 \\
\hline Arbutus arizonica & (A. Gray) Sarg. & 0.88575 & 0.93412 & 0.88295 \\
\hline Arbutus madrensis & M. González & 0.89143 & 0.90645 & 0.90351 \\
\hline Arbutus occidentalis & McVaugh \& Rosatti & 0.92219 & 0.91235 & 0.84993 \\
\hline Arbutus tessellata & P.D. Sorensen & 0.94684 & 0.94257 & 0.90476 \\
\hline Arbutus xalapensis & Kunth & 0.90975 & 0.92770 & 0.87568 \\
\hline Arctostaphylos pungens & Kunth & 0.94028 & 0.93060 & 0.89945 \\
\hline Aspidosperma desmanthum & Benth. ex Müll. Arg. & 0.97772 & 0.98071 & 0.95055 \\
\hline Avicennia germinans & (L.) L. & 0.89935 & 0.89812 & 0.89871 \\
\hline Baccharis conferta & Kunth & 0.97185 & 0.95683 & 0.92997 \\
\hline Baccharis heterophylla & Kunth & 0.88627 & 0.87031 & 0.84503 \\
\hline Beaucarnea pliabilis & (Baker) Rose & 0.38253 & 0.83880 & 0.91108 \\
\hline Beilschmiedia manantlanensis & Cuevas \& Cochrane & 0.98680 & 0.98010 & 0.99394 \\
\hline Beilschmiedia mexicana & (Mez) Kosterm. & 0.84786 & 0.88891 & 0.75602 \\
\hline Beilschmiedia ovalioides & Sach. Nishida & 0.87682 & 0.95186 & 0.91304 \\
\hline Bellucia grossularioides & (L.) Triana & 0.94606 & 0.94389 & 0.96253 \\
\hline Bernardia dodecandra & (Sessé ex Cavagnaro, David) McVaugh & 0.90375 & 0.90599 & 0.93254 \\
\hline Blakea purpusii & Brandegee & 0.79061 & 0.87483 & 0.89664 \\
\hline Bocconia glaucifolia & Hutch. & 0.77744 & 0.75725 & 0.80134 \\
\hline Boehmeria ulmifolia & Wedd. & 0.93252 & 0.92835 & 0.89086 \\
\hline Bourreria mollis & Standl. & 0.95688 & 0.91239 & 0.91699 \\
\hline Bunchosia sonorensis & Rose & 0.97929 & 0.93759 & 0.99531 \\
\hline Bursera bicolor & (Willd. ex Schltdl.) Engl. & 0.70936 & 0.69755 & 0.89668 \\
\hline Bursera bipinnata & (DC.) Engl. & 0.86089 & 0.84073 & 0.88425 \\
\hline Bursera copallifera & (DC.) Bullock & 0.85907 & 0.84218 & 0.92209 \\
\hline Bursera coyucensis & Bullock & 0.99531 & 0.89704 & 0.98252 \\
\hline Bursera denticulata & McVaugh \& Rzed. & 0.94730 & 0.93672 & 0.95450 \\
\hline Bursera discolor & Rzed. & 0.69330 & 0.67771 & 0.85271 \\
\hline Bursera diversifolia & Rose & 0.88323 & 0.91163 & 0.94240 \\
\hline Bursera excelsa & (Kunth) Engl. & 0.82716 & 0.71271 & 0.93003 \\
\hline Bursera heliae & Rzed. \& Calderón & 0.36198 & 0.84415 & 0.94968 \\
\hline Bursera instabilis & McVaugh \& Rzed. & 0.99625 & 0.98754 & 0.99696 \\
\hline Bursera kerberi & Engl. & 0.87562 & 0.91627 & 0.94693 \\
\hline Bursera multifolia & (Rose) Engl. & 0.88516 & 0.76603 & 0.84725 \\
\hline Bursera multijuga & Engl. & 0.86322 & 0.92754 & 0.96918 \\
\hline Bursera sarukhanii & Guevara \& Rzed. & 0.90228 & 0.92210 & 0.93168 \\
\hline
\end{tabular}


Anexo 1. Continuación

\begin{tabular}{|c|c|c|c|c|}
\hline Especie & Autoridad & GAM_T & GAM_P & GAM_DH ${ }_{\text {anual }}$ \\
\hline Bursera stenophylla & Sprague \& L. Riley & 0.80398 & 0.93910 & 0.94872 \\
\hline Bursera trifoliolata & Bullock & 0.98258 & 0.92987 & 0.93095 \\
\hline Bursera vejar-vazquezii & Miranda & 0.16081 & 0.58443 & 0.87014 \\
\hline Caesalpinia acapulcensis & Standl. & 0.86780 & 0.98115 & 0.96278 \\
\hline Caesalpinia caladenia & Standl. & 0.86929 & 0.80259 & 0.85241 \\
\hline Caesalpinia coccinea & G.P. Lewis \& J.L. Contr. & 0.98518 & 0.99084 & 1.00000 \\
\hline Caesalpinia gaumeri & Greenm. & 0.54164 & 0.80046 & 0.88290 \\
\hline Caesalpinia pannosa & Brandegee & 0.94534 & 0.89584 & 0.96049 \\
\hline Caesalpinia platyloba & (S. Watson) N. Zamora & 0.91518 & 0.90702 & 0.88254 \\
\hline Caesalpinia standleyi & (Britton \& Rose) Standl. & 0.87321 & 0.95506 & 0.99157 \\
\hline Calliandra laevis & Rose & 0.96528 & 0.95262 & 0.99278 \\
\hline Calyptranthes karlingii & Standl. & 0.87494 & 0.87407 & 0.68292 \\
\hline Cameraria latifolia & L. & 0.50543 & 0.71709 & 0.62083 \\
\hline Cascabela thevetioides & (Kunth) Lippold & 0.68262 & 0.90057 & 0.98011 \\
\hline Casimiroa greggii & (S. Watson) F. Chiang & 0.89180 & 0.90590 & 0.89579 \\
\hline Casimiroa pringlei & (S. Watson) Engl. ex Engl. \& Prantl & 0.94452 & 0.91029 & 0.84497 \\
\hline Cecropia angustifolia & Trécul & 0.91905 & 0.96307 & 0.95241 \\
\hline Celastrus vulcanicolus & Donn. Sm. & 0.90458 & 0.94085 & 0.49630 \\
\hline Cephalanthus occidentalis & $\mathrm{L}$ & 0.77486 & 0.60241 & 0.81624 \\
\hline Cephalocereus columna-trajani & (Karw. ex Pfeiff.) K. Schum. & 0.58302 & 0.51362 & 0.94490 \\
\hline Cephalocereus totolapensis & (Bravo \& T. MacDoug.) Buxb. & 0.52521 & 0.73607 & 0.94763 \\
\hline Cestrum nitidum & M. Martens \& Galeotti & 0.87230 & 0.86171 & 0.76694 \\
\hline Cestrum tomentosum & L.f. & 0.84048 & 0.88340 & 0.84376 \\
\hline Chiranthodendron pentadactylon & Larreat. & 0.84479 & 0.83207 & 0.87410 \\
\hline Chrysophyllum venezuelanense & (Pierre) T.D. Penn. & 0.94056 & 0.95023 & 0.93692 \\
\hline Cinnamomum concinnum & Lorea-Hern. & 0.69521 & 0.79536 & 0.92163 \\
\hline Cinnamomum effusum & (Meisn.) Kosterm. & 0.84283 & 0.90433 & 0.94241 \\
\hline Citharexylum steyermarkii & Moldenke & 0.88368 & 0.91233 & 0.92452 \\
\hline Clethra alcoceri & Greenm. & 0.90507 & 0.83925 & 0.79653 \\
\hline Clethra luzmariae & L.M. González & 0.95915 & 0.97108 & 0.97108 \\
\hline Clethra oleoides & L.O. Williams & 0.93156 & 0.92521 & 0.75100 \\
\hline Clethra rosei & Britton & 0.76154 & 0.87267 & 0.95848 \\
\hline Cleyera integrifolia & (Benth.) Choisy & 0.86717 & 0.94609 & 0.84786 \\
\hline Cleyera theoides & (Sw.) Choisy & 0.97265 & 0.96472 & 0.90248 \\
\hline Clusia salvinii & Donn. Sm. & 0.72734 & 0.71114 & 0.60904 \\
\hline Cnidoscolus megacanthus & Breckon & 0.95385 & 0.97822 & 0.96770 \\
\hline Cnidoscolus spinosus & Lundell & 0.99477 & 0.97683 & 0.99653 \\
\hline Cnidoscolus tepiquensis & (Cost. \& Gall.) McVaugh & 0.90493 & 0.95152 & 0.97939 \\
\hline Coccoloba belizensis & Standl. & 0.92526 & 0.91156 & 0.87716 \\
\hline Cojoba arborea & (L.) Britton \& Rose & 0.93032 & 0.91976 & 0.81047 \\
\hline Comarostaphylis arbutoides & Lindl. & 0.97401 & 0.98237 & 0.97882 \\
\hline Comarostaphylis sharpii & Dorr \& Diggs & 0.92028 & 0.96000 & 0.85872 \\
\hline Conostegia volcanalis & Standl. \& Steyerm. & 0.96779 & 0.96563 & 0.96495 \\
\hline Cordia morelosana & Standl. & 0.84755 & 0.84588 & 0.85706 \\
\hline Cordia stellata & Greenm. & 0.86985 & 0.65940 & 0.91181 \\
\hline Cosmibuena matudae & (Standl.) L.O. Williams & 0.76908 & 0.75192 & 0.80619 \\
\hline
\end{tabular}


Anexo 1. Continuación

\begin{tabular}{|c|c|c|c|c|}
\hline Especie & Autoridad & GAM_T & GAM_P ${ }_{\text {anual }}$ & GAM_DH ${ }_{\text {anual }}$ \\
\hline Critoniopsis leiocarpa & (DC.) H. Rob. & 0.56492 & 0.63086 & 0.80270 \\
\hline Critoniopsis tomentosa & DC. & 0.89661 & 0.87721 & 0.81219 \\
\hline Croton billbergianus & Müll. Arg. & 0.92434 & 0.94966 & 0.89980 \\
\hline Croton dioicum & Cav. & 0.92942 & 0.79000 & 0.95807 \\
\hline Croton rosarianus & Mart. Gord. \& Cruz Durán & 0.99375 & 0.95479 & 1.00000 \\
\hline Ctenardisia purpusii & (Brandegee) Lundell & 0.12376 & 0.86101 & 0.89892 \\
\hline Cymbopetalum baillonii & R.E. Fr. & 0.94583 & 0.98589 & 0.98782 \\
\hline Cynometra retusa & Britton \& Rose & 0.98178 & 0.98858 & 0.98515 \\
\hline Cyrtocarpa edulis & (Brandegee) Standl. & 0.80319 & 0.79167 & 0.97356 \\
\hline Cyrtocarpa procera & Kunth & 0.66634 & 0.74022 & 0.89773 \\
\hline Dalbergia palo-escrito & Rzed. \& Guridi-Gómez & 0.96770 & 0.98294 & 0.64485 \\
\hline Dalea capitata & (Rydb.) Barneby & 0.90820 & 0.77063 & 0.86016 \\
\hline Dalea leucostachya & A. Gray & 0.78327 & 0.83916 & 0.90682 \\
\hline Daphnopsis tuerckheimiana & Donn. Sm. & 0.40867 & 0.48152 & 0.80870 \\
\hline Dasylirion cedrosanum & Trel. & 0.95055 & 0.64972 & 0.90682 \\
\hline Dendropanax arboreus & (L.) Decne. \& Planch. & 0.54159 & 0.61259 & 0.87018 \\
\hline Dendropanax pallidus & M.J. Cannon \& Cannon & 0.52016 & 0.66757 & 0.79410 \\
\hline Deppea grandiflora & Schltdl. & 0.90612 & 0.73174 & 0.89625 \\
\hline Desmopsis mexicana & R.E. Fr. & 0.98889 & 0.99399 & 0.97598 \\
\hline Dialium guianense & (Aubl.) Sandwith & 0.97733 & 0.98171 & 0.96753 \\
\hline Diospyros sinaloensis & S.F. Blake & 0.93702 & 0.88773 & 0.92862 \\
\hline Drimys granadensis & L. f. & 0.98572 & 0.97476 & 0.85103 \\
\hline Dussia cuscatlanica & (Standl.) Standl. \& Steyerm. & 0.84490 & 0.74934 & 0.89083 \\
\hline Ehretia latifolia & Loisel. & 0.91904 & 0.79890 & 0.81618 \\
\hline Erythrina breviflora & Sessé \& Moc. ex DC. & 0.87303 & 0.86035 & 0.77962 \\
\hline Esenbeckia hartmanii & Robinson \& Fernald & 0.82128 & 0.82281 & 0.98675 \\
\hline Eugenia citroides & Lundell & 0.54762 & 0.62897 & 0.73264 \\
\hline Eugenia culminicola & McVaugh & 0.90204 & 0.83833 & 0.93352 \\
\hline Eugenia laevis & O. Berg & 0.57653 & 0.88058 & 0.83945 \\
\hline Eugenia oerstediana & O. Berg & 0.85122 & 0.89181 & 0.86280 \\
\hline Eugenia ravenii & Lundell & 0.31960 & 0.97787 & 0.99282 \\
\hline Eugenia tonii & Lundell & 0.94350 & 0.93390 & 0.94655 \\
\hline Euphorbia antisyphilitica & Zucc. & 0.88649 & 0.69132 & 0.91956 \\
\hline Euphorbia sinclairiana & Benth. & 0.52338 & 0.69406 & 0.80011 \\
\hline Exothea diphylla & (Standl.) Lundell & 0.80321 & 0.92717 & 0.96898 \\
\hline Eysenhardtia orthocarpa & (A. Gray) S. Watson & 0.63843 & 0.73478 & 0.80046 \\
\hline Faramea occidentalis & (L.) A. Rich. & 0.93051 & 0.93689 & 0.81581 \\
\hline Ficus apollinaris & Dugand & 0.92767 & 0.97287 & 0.94306 \\
\hline Ficus fuscescens & (Liebm.) Miq. & 0.32268 & 0.39548 & 0.96610 \\
\hline Ficus membranacea & C. Wright & 0.93800 & 0.93505 & 0.93505 \\
\hline Ficus microchlamys & Humb. \& Bonpl. ex Willd. & 0.70283 & 0.83389 & 0.91514 \\
\hline Flourensia cernua & DC. & 0.92859 & 0.75847 & 0.96521 \\
\hline Flourensia laurifolia & DC. & 0.94862 & 0.90203 & 0.95695 \\
\hline Forchhammeria watsonii & Rose & 0.87156 & 0.72727 & 0.88154 \\
\hline Fouquieria formosa & Kunth & 0.92315 & 0.95874 & 0.96815 \\
\hline Fouquieria splendens & Engelm. & 0.88476 & 0.92530 & 0.93050 \\
\hline
\end{tabular}


Anexo 1. Continuación

\begin{tabular}{|c|c|c|c|c|}
\hline Especie & Autoridad & GAM_T & GAM_P $\mathbf{P}_{\text {anual }}$ & GAM_DH ${ }_{\text {anual }}$ \\
\hline Fuchsia arborescens & Sims & 0.91819 & 0.90868 & 0.81539 \\
\hline Fuchsia paniculata & Lindl. & 0.97977 & 0.96104 & 0.96249 \\
\hline Gaussia maya & (O.F. Cook) H.J. Quero R. & 0.97635 & 0.97027 & 0.98042 \\
\hline Gochnatia arborescens & Brandegee & 0.95163 & 0.89110 & 0.99432 \\
\hline Guarea glabra & Vahl & 0.91424 & 0.92753 & 0.92122 \\
\hline Gyrocarpus mocinoi & Espejo & 0.87187 & 0.68301 & 0.92614 \\
\hline Hamelia xorullensis & Kunth & 0.91930 & 0.86716 & 0.93764 \\
\hline Hampea breedlovei & Fryxell & 0.69815 & 0.86580 & 0.89781 \\
\hline Hampea montebellensis & Fryxell & 0.53937 & 0.95402 & 0.70498 \\
\hline Hampea nutricia & Fryxell & 0.96275 & 0.97449 & 0.96865 \\
\hline Hampea tomentosa & (C. Presl) Standl. & 0.96878 & 0.97275 & 0.98214 \\
\hline Harpalyce formosa & DC. & 0.27708 & 0.57441 & 0.84325 \\
\hline Harpalyce rupicola & Donn. Sm. & 0.74510 & 0.80482 & 0.87939 \\
\hline Hasseltia guatemalensis & Warb. & 0.95133 & 0.96110 & 0.97464 \\
\hline Helicteres baruensis & Jacq. & 0.80646 & 0.78221 & 0.84986 \\
\hline Heliocarpus occidentalis & Rose & 0.84315 & 0.84571 & 0.92523 \\
\hline Heliocarpus palmeri & S. Watson & 0.82546 & 0.97467 & 0.96923 \\
\hline Hirtella racemosa & Lam. & 0.90232 & 0.94135 & 0.92875 \\
\hline Hoffmannia nicotianifolia & (M. Martens \& Galeotti) L.O. Williams & 0.91087 & 0.93243 & 0.76373 \\
\hline Ilex condensata & Turcz. & 0.76450 & 0.87020 & 0.86213 \\
\hline Ilex costaricensis & Donn. Sm. & 0.91883 & 0.94318 & 0.92582 \\
\hline Ilex rubra & S. Watson & 0.76898 & 0.89687 & 0.89874 \\
\hline Indigofera palmeri & S. Watson & 0.88570 & 0.83920 & 0.89715 \\
\hline Inga huastecana & M. Sousa & 0.99266 & 0.96327 & 0.92028 \\
\hline Inga jinicuil & Schltdl. & 0.90439 & 0.91621 & 0.94431 \\
\hline Inga sapindoides & Willd. & 0.35223 & 0.63231 & 0.86139 \\
\hline Jatropha cardiophylla & (Torr.) Müll. Arg. & 0.72457 & 0.69020 & 0.88830 \\
\hline Jatropha vernicosa & Brandegee & 0.82661 & 0.73508 & 0.92804 \\
\hline Juglans mollis & Engelm. & 0.92649 & 0.83198 & 0.77744 \\
\hline Juniperus blancoi & Martínez & 0.99028 & 0.98476 & 0.91683 \\
\hline Juniperus californica & Carrière & 0.81764 & 0.59613 & 0.96559 \\
\hline Juniperus durangensis & Martínez & 0.77570 & 0.88035 & 0.84053 \\
\hline Juniperus jaliscana & Martínez & 0.98024 & 0.96618 & 0.88455 \\
\hline Karwinskia calderonii & Standl. & 0.92099 & 0.92839 & 0.94937 \\
\hline Koanophyllon pittieri & (Klatt) R.M. King \& H. Robinson & 0.68075 & 0.73938 & 0.70796 \\
\hline Laetia thamnia & L. & 0.91360 & 0.43032 & 0.91417 \\
\hline Lafoensia punicifolia & DC. & 0.71409 & 0.71637 & 0.95541 \\
\hline Leandra subseriata & (Naudin) Cogn. & 0.94793 & 0.97007 & 0.92761 \\
\hline Lecointea amazonica & Ducke & 0.13443 & 0.82168 & 0.81935 \\
\hline Licania arborea & Seem. & 0.54085 & 0.48710 & 0.81857 \\
\hline Licania hypoleuca & Benth. & 0.95133 & 0.96235 & 0.83696 \\
\hline Licaria chinanteca & Lorea-Hern. & 0.33623 & 0.65897 & 0.75000 \\
\hline Liquidambar styraciflua & L. & 0.91192 & 0.92778 & 0.82194 \\
\hline Lonchocarpus caudatus & Pittier & 0.73712 & 0.73055 & 0.89248 \\
\hline Lonchocarpus hondurensis & Benth. & 0.93156 & 0.95359 & 0.86992 \\
\hline Magnolia iltisiana & A. Vázquez & 0.98279 & 0.94222 & 0.96294 \\
\hline
\end{tabular}


Anexo 1. Continuación

\begin{tabular}{|c|c|c|c|c|}
\hline Especie & Autoridad & GAM_T & GAM_P & GAM_DH ${ }_{\text {anual }}$ \\
\hline Malpighia mexicana & A. Juss. & 0.57341 & 0.71392 & 0.90241 \\
\hline Matayba oppositifolia & (A. Rich.) Britton & 0.53770 & 0.54979 & 0.90683 \\
\hline Miconia glabrata & Cogn. & 0.19260 & 0.53973 & 0.99726 \\
\hline Miconia hondurensis & Donn. Sm. & 0.30185 & 0.43931 & 0.96308 \\
\hline Miconia impetiolaris & (Sw.) D. Don ex DC. & 0.36082 & 0.48614 & 0.83030 \\
\hline Miconia minutiflora & (Bonpl.) DC. & 0.83195 & 0.55580 & 0.91312 \\
\hline Mimosa distachya & Cav. & 0.68736 & 0.69194 & 0.87370 \\
\hline Mimosa eurycarpa & B.L. Rob. & 0.93806 & 0.94730 & 0.93016 \\
\hline Mimosa lactiflua & Delile ex Benth. & 0.87790 & 0.76891 & 0.86773 \\
\hline Mimosa palmeri & Rose & 0.73216 & 0.86222 & 0.87106 \\
\hline Nectandra ambigens & (S.F. Blake) C.K. Allen & 0.93218 & 0.95314 & 0.97010 \\
\hline Nectandra leucocome & Rohwer & 0.97217 & 0.98177 & 0.99475 \\
\hline Nectandra lundellii & C.K. Allen & 0.70897 & 0.70300 & 0.80325 \\
\hline Oreopanax echinops & (Cham. \& Schltdl.) Decne. \& Planch. & 0.96007 & 0.96317 & 0.94196 \\
\hline Oreopanax flaccidus & Marchal & 0.93448 & 0.96729 & 0.97155 \\
\hline Oreopanax xalapensis & (Kunth) Decne. \& Planch. & 0.89205 & 0.89783 & 0.84808 \\
\hline Parkinsonia praecox & (Ruiz \& Pav. ex Hook.) Hawkins & 0.75951 & 0.80809 & 0.87850 \\
\hline Pinus ayacahuite & C. Ehrenb. ex Schltdl. & 0.91854 & 0.91202 & 0.89219 \\
\hline Pinus devoniana & Lindl. & 0.89593 & 0.92086 & 0.87631 \\
\hline Pinus greggii & Engelm. ex Parl. & 0.91330 & 0.88374 & 0.87427 \\
\hline Pinus hartwegii & Lindl. & 0.95359 & 0.90105 & 0.90677 \\
\hline Pinus leiophylla & Schiede ex Schltdl. \& Cham. & 0.91106 & 0.95118 & 0.93177 \\
\hline Pinus maximinoi & H.E. Moore & 0.80296 & 0.89667 & 0.81488 \\
\hline Pinus oocarpa & Schiede ex Schltdl. & 0.59951 & 0.82605 & 0.88834 \\
\hline Pinus pseudostrobus & Lindl. & 0.90582 & 0.88186 & 0.76946 \\
\hline Pinus tecunumanii & F. Schwerdtf. ex Eguiluz \& J.P.Perry & 0.62018 & 0.60726 & 0.73927 \\
\hline Pinus teocote & Schltdl. \& Cham. & 0.92935 & 0.92677 & 0.90190 \\
\hline Pterocarpus acapulcensis & Rose & 0.93843 & 0.90863 & 0.95348 \\
\hline Pterocarpus orbiculatus & DC. & 0.90808 & 0.90901 & 0.93679 \\
\hline Quercus acutifolia & Née & 0.88106 & 0.82869 & 0.83335 \\
\hline Quercus benthamii & A. DC. & 0.89744 & 0.89236 & 0.94081 \\
\hline Quercus castanea & Née & 0.88150 & 0.89851 & 0.89428 \\
\hline Quercus conspersa & Benth. & 0.85118 & 0.85847 & 0.84982 \\
\hline Quercus crassifolia & Bonpl. & 0.88621 & 0.93426 & 0.92032 \\
\hline Quercus crassipes & Bonpl. & 0.94623 & 0.94758 & 0.91790 \\
\hline Quercus depressipes & Trel. & 0.88316 & 0.90568 & 0.88221 \\
\hline Quercus durifolia & Seemen & 0.91912 & 0.84314 & 0.87369 \\
\hline Quercus elliptica & Née & 0.87670 & 0.91480 & 0.95190 \\
\hline Quercus emoryi & Torr. & 0.79794 & 0.82109 & 0.82228 \\
\hline Quercus eugeniifolia & Née & 0.91379 & 0.83140 & 0.85707 \\
\hline Quercus gentryi & C.H. Mull. & 0.74153 & 0.76783 & 0.70862 \\
\hline Quercus greggii & (A. DC.) Trel. & 0.95290 & 0.91601 & 0.94541 \\
\hline Quercus hintoniorum & Nixon \& C.H. Mull. & 0.84515 & 0.91049 & 0.94311 \\
\hline Quercus hypoleucoides & A. Camus & 0.94707 & 0.89881 & 0.90612 \\
\hline Quercus hypoxantha & Trel. & 0.93921 & 0.88210 & 0.88841 \\
\hline Quercus insignis & M. Martens \& Galeotti & 0.90543 & 0.85119 & 0.87210 \\
\hline
\end{tabular}


Anexo 1. Continuación

\begin{tabular}{llccc}
\hline Especie & Autoridad & GAM_T & GAM_P anual & GAM_DH \\
\hline Quercus laeta & Liebm. & 0.90870 & 0.91075 & 0.89540 \\
Quercus laurina & Bonpl. & 0.90828 & 0.88766 & 0.71105 \\
Quercus liebmannii & Oerst. ex Trel. & 0.77368 & 0.71477 & 0.90483 \\
Quercus magnoliifolia & Née & 0.77869 & 0.79649 & 0.87725 \\
Quercus martinezii & C.H. Mull. & 0.81064 & 0.81553 & 0.87610 \\
Quercus mexicana & Bonpl. & 0.94529 & 0.94124 & 0.82636 \\
Quercus obtusata & Bonpl. & 0.90979 & 0.91708 & 0.91210 \\
Quercus peduncularis & Née & 0.42998 & 0.63565 & 0.84327 \\
Quercus pringlei & Seemen & 0.91712 & 0.82536 & 0.84134 \\
Quercus repanda & Bonpl. & 0.97570 & 0.97854 & 0.93048 \\
Quercus resinosa & Liebm. & 0.90351 & 0.91108 & 0.91163 \\
Quercus rugosa & Née & 0.94795 & 0.94539 & 0.90320 \\
Quercus saltillensis & Trel. & 0.97056 & 0.92676 & 0.88546 \\
Quercus scytophylla & Liebm. & 0.82958 & 0.88985 & 0.90505 \\
Quercus splendens & Née & 0.87622 & 0.82702 & 0.75120 \\
Quercus striatula & Trel. & 0.95235 & 0.89051 & 0.94582 \\
Quercus subspathulata & Trel. & 0.81622 & 0.81562 & 0.79871 \\
Quercus tuberculata & Liebm. & 0.58584 & 0.69898 & 0.85975 \\
Quercus turbinella & Greene & 0.87717 & 0.94505 & 0.87882 \\
Quercus urbani & Trel. & 0.67637 & 0.62139 & 0.89111 \\
Quercus uxoris & McVaugh & 0.55793 & 0.58135 & 0.84895 \\
Quercus viminea & Trel. & 0.76293 & 0.88256 & 0.87658 \\
Sebastiania cornuta & (Lundell) A. L. Melo \& Esser & 0.40532 & 0.36108 & 0.92081 \\
Stemmadenia pubescens & Benth. & 0.63630 & 0.72810 & 0.85803 \\
Zanthoxylum arborescens & Rose & 0.87933 & 0.87614 & 0.93998 \\
Zanthoxylum mollissimum & (Engl.) P. Wilson & 0.54872 & 0.70196 & 0.83501 \\
\hline
\end{tabular}

